\title{
Predictors of Inappropriate Use of Diagnostic Tests and Management of Bronchiolitis
}

\author{
Lorena Sarmiento, ${ }^{1}$ Gladys E. Rojas-Soto, ${ }^{2}$ and Carlos E. Rodríguez-Martínez ${ }^{3,4}$ \\ ${ }^{1}$ Department of Pediatrics, Clinica Universitaria Colombia, Fundacion Universitaria Sanitas, Bogota, Colombia \\ ${ }^{2}$ Department of Pediatric Respiratory Medicine, Hospital El Pino, University of Santiago de Chile (USACH), Santiago, Chile \\ ${ }^{3}$ Department of Pediatrics, School of Medicine, Universidad Nacional de Colombia, Bogota, Colombia \\ ${ }^{4}$ Department of Pediatric Pulmonology and Pediatric Critical Care Medicine, School of Medicine, \\ Universidad El Bosque, Bogota, Colombia
}

Correspondence should be addressed to Carlos E. Rodríguez-Martínez; carerodriguezmar@unal.edu.co

Received 19 February 2017; Accepted 28 May 2017; Published 3 July 2017

Academic Editor: Luis Martinez-Sobrido

Copyright ( 2017 Lorena Sarmiento et al. This is an open access article distributed under the Creative Commons Attribution License, which permits unrestricted use, distribution, and reproduction in any medium, provided the original work is properly cited.

\begin{abstract}
Background. The aim of the present study was to determine predictors of inappropriate use of diagnostic tests and management of bronchiolitis in a population of hospitalized infants. Methods. In an analytical cross-sectional study, we determined independent predictors of the inappropriate use of diagnostic tests and management of bronchiolitis in a population of hospitalized infants. We defined a composite outcome score as the main outcome variable. Results. Of the 303 included patients, 216 (71.3\%) experienced an inappropriate use of diagnostic tests and treatment of bronchiolitis. After controlling for potential confounders, it was found that atopic dermatitis (OR 5.30; CI 95\% 1.14-24.79; $p=0.034$ ), length of hospital stay (OR 1.48; CI 95\% 1.08-2.03; $p=0.015$ ), and the number of siblings (OR 1.92; CI 95\% 1.13-3.26; $p=0.015$ ) were independent predictors of an inappropriate use of diagnostic tests and treatment of the disease. Conclusions. Inappropriate use of diagnostic tests and treatment of bronchiolitis was a highly prevalent outcome in our population of study. Participants with atopic dermatitis, a longer hospital stay, and a greater number of siblings were at increased risk for inappropriate use of diagnostic tests and management of the disease.
\end{abstract}

\section{Introduction}

Acute bronchiolitis represents the most important cause of lower respiratory tract infection during the first year of life and is the leading reason for hospitalization of infants beyond the neonatal period [1]. The disease is usually associated with substantial direct and indirect costs, not only for healthcare systems but also for families and society as a whole [2]. Although bronchiolitis is an extremely common disease and several clinical practice guidelines (CPGs) of acceptable quality have been developed [3], there is still a significant use and overuse of medications with insufficient evidence of effectiveness [4] and use of unnecessary diagnostic tests [5], generating unnecessary and costly resource use with no improvement in important clinical outcomes [6]. It is important to identify predictors of inappropriate use of diagnostic tests and management of bronchiolitis, which would help to plan more focused strategies and more targeted efforts in hope of achieving better disease management. An implementation strategy of CPGs that deal with the identified predictors could help to reduce the inappropriate use and overuse of diagnostic tests and medications that lack strong evidence that supports a recommendation for their routine use [4]. This implementation strategy could lead to the economical and effective use of health care services and the avoidance of wasting resources without reducing the quality of these services, finally contributing to the improvement of disease outcomes. To date, only a few studies have identified predictors of inappropriate use of diagnostic tests and management of bronchiolitis, showing that the region or the community where the patients were treated $[7,8]$ and whether patients were treated in a non-universityaffiliated hospital were independent predictors of inappropriate management of bronchiolitis [7]. However, there is a need 
for further studies, which ideally should account for patientrelated variables such as disease severity and the presence of comorbidities and atopic conditions, not only in patients but also in their families. Accordingly, the aim of the present study was to identify potential factors associated with the inappropriate use of diagnostic tests and management of bronchiolitis in a population of infants hospitalized for acute viral bronchiolitis in the Fundación Hospital La Misericordia, a university-affiliated hospital located in the metropolitan area of Bogota, Colombia.

\section{Material and Methods}

2.1. Bronchiolitis Clinical Practice Guideline (CPG). At the time of the study's inception, an evidence-based bronchiolitis institutional CPG was available at the Fundación Hospital La Misericordia and served as a basis for guidance about which diagnostic tests and medications should be used in infants treated in the hospital for any acute viral bronchiolitis episode [9].

The bronchiolitis CPG recommends against routine use of diagnostic tests such as a hemogram, C-reactive protein, and procalcitonin and suggests that C-reactive protein and procalcitonin should be ordered only for infants with suspected serious bacterial infections. Likewise, the CPG recommends against the routine use of chest $\mathrm{X}$-rays, suggesting that they should be ordered only for infants with diagnostic uncertainty, severe disease, or an atypical disease course. With respect to pharmacologic therapy, the CPG also recommends against the routine use of inhaled bronchodilators (beta 2 agonists, anticholinergics), nebulized epinephrine, and anti-inflammatories (inhaled or systemic corticosteroids) but allows for the option of a monitored trial of inhaled bronchodilators or nebulized epinephrine, continuing their administration only if there is a documented positive clinical response to the trial using an objective means of evaluation. Finally, the CPG also recommends that nebulized hypertonic saline be administered to infants hospitalized for bronchiolitis in order to shorten hospital stay but recommends against its use in the emergency department.

2.2. Study Design and Procedures. An analytical crosssectional study was performed during the period from March to June 2014, in a random sample of patients aged $<2$ years hospitalized in the Fundacion Hospital de La Misericordia with a diagnosis of acute bronchiolitis (ICD-10 codes J21, J21.0, J21.1, J21.8, and J21.9). We chose this time period because it roughly coincides with the main bronchiolitis season in the city [10]. After reviewing the electronic medical records (EMRs), we collected the following demographic and clinical information: date of admission, age, gender, occurrence of breastfeeding for at least six months, nursery attendance, number of siblings, number of days with respiratory symptoms, presence of underlying disease conditions, previous use of oxygen therapy, personal history or diagnosis of atopic dermatitis, and parental history of asthma. Likewise, we collected information related to the use of diagnostic tests and use of pharmacologic therapy when diagnosing and/or treating bronchiolitis. Finally, we collected information related to the care or the disease-severity parameters, such as length of hospital stay, need for home oxygen administration, need for pediatric intensive care unit (PICU) admission, need for endotracheal intubation, and mortality.

The study protocol was approved by the local ethics board.

2.3. Outcome Definition. As the primary outcome of interest, we defined a priori a composite outcome score, which we termed "inappropriate use of diagnostic tests and treatment of bronchiolitis." This composite outcome score aggregated the use of diagnostic tests (hemogram, C-reactive protein, procalcitonin, chest radiography, and tests for detection of respiratory viruses) and the use of pharmacologic therapy (inhaled or nebulized beta 2 agonists, inhaled or nebulized anticholinergics, nebulized epinephrine, inhaled corticosteroids, systemic corticosteroids, antibiotics, and nebulized hypertonic saline). Each of these 12 items had a binary score: 0 indicating that the diagnostic test or the medication was not used and 1 indicating that they were used (except for the use of nebulized hypertonic saline, which also had a binary score, but with 0 indicating that the medication was used and 1 indicating that it was not used). The use of Creactive protein and procalcitonin was deemed as appropriate in patients with suspected or confirmed serious bacterial infections, the use of chest X-rays was deemed as appropriate in patients with severe disease, or with atypical disease course, and the use of inhaled beta 2 agonists and epinephrine was deemed as appropriate only if they were continued after a positive clinical response to a monitored trial. The total score was calculated by adding up the scores for the 12 items, resulting in a total score ranging from 0 to 12 (best to worst). A composite outcome score of $>3$ was designated as "inappropriate use of diagnostic tests and treatment of bronchiolitis." The sole use of anticholinergics (nebulized or inhaled) or corticosteroids (inhaled or systemic) was deemed to be an "inappropriate use of diagnostic tests and treatment of bronchiolitis," independent of the composite outcome score. A sensitivity analysis was performed using a range of cutoff values from 1 to 5 of the composite outcome score for defining "inappropriate use of diagnostic tests and treatment of bronchiolitis."

2.4. Statistical Analysis. Continuous variables are presented as mean \pm standard deviation (SD) or median (interquartile range $[\mathrm{IQR}]$ ), whichever is appropriate. Categorical variables are presented as numbers (percentage). Differences between continuous variables were analyzed using the unpaired $t$-test or Wilcoxon's signed rank test, whichever was appropriate. Associations between categorical variables and the outcome variable were analyzed using the chi-square test or Fisher's exact test, whichever was appropriate. To identify factors independently associated with an inappropriate use of diagnostic tests and treatment of bronchiolitis, we used logistic regression models, adjusting for potential confounding variables. The predictive variables included in the models were the age of the patients, the number of days with respiratory symptoms, a parental history of asthma, and the presence of at least one underlying disease or condition associated with severe disease. All statistical tests were two-tailed, and the 
TABLE 1: Demographic characteristics and clinical information of the patients included in the study, according to the appropriateness of bronchiolitis diagnosis and treatment.

\begin{tabular}{|c|c|c|c|}
\hline Variable & $\begin{array}{l}\text { Patients with inadequate } \\
\text { diagnosis and treatment } \\
(n=216)\end{array}$ & $\begin{array}{l}\text { Patients with adequate } \\
\text { diagnosis and treatment } \\
(n=87)\end{array}$ & $p$ value \\
\hline Age (months; median (interquartile range-IQR)) & $3.0(1.0-7.0)$ & $3.0(1.0-6.0)$ & 0.966 \\
\hline Gender, M/F & $131 / 85$ & $45 / 42$ & 0.154 \\
\hline Presence of breastfeeding for at least six months & $31(14.4 \%)$ & $14(16.1 \%)$ & 0.665 \\
\hline Nursery attendance & $20(9.3 \%)$ & $7(8.0 \%)$ & 0.780 \\
\hline Number of siblings & $1.0(0.0-1.0)$ & $0.0(0.0-1.0)$ & 0.306 \\
\hline \multicolumn{4}{|l|}{ Number of days with respiratory symptoms } \\
\hline Less than 24 hours & $19(8.8 \%)$ & $12(13.8 \%)$ & 0.211 \\
\hline Between 24 and 72 hours & $192(88.8 \%)$ & $71(81.6 \%)$ & 0.095 \\
\hline More than 72 hours & $3(1.4 \%)$ & $1(1.1 \%)$ & 1.00 \\
\hline Presence of underlying disease conditions & $26(12.0 \%)$ & $13(14.9 \%)$ & 0.513 \\
\hline Previous use of oxygen therapy & $6.0(6.9 \%)$ & $5.0(2.3 \%)$ & 0.055 \\
\hline Personal history or diagnosis of atopic dermatitis & $7.0(8.0 \%)$ & $6.0(2.8 \%)$ & 0.073 \\
\hline Parental history of asthma & $32(14.8 \%)$ & $13(14.9 \%)$ & 0.846 \\
\hline Respiratory syncytial virus isolation & $95(44.0 \%)$ & $20(23.0 \%)$ & $<0.001$ \\
\hline Length of hospital stay & $4.0(3.0-6.0)$ & $3.0(2.0-4.0)$ & $<0.001$ \\
\hline Need for home oxygen administration & $76(35.2 \%)$ & $22(25.3 \%)$ & 0.082 \\
\hline Need for pediatric intensive care unit & $4(1.9 \%)$ & $0(0.0 \%)$ & 0.675 \\
\hline Need for endotracheal intubation & $1(0.5 \%)$ & $0(0.0 \%)$ & 1.00 \\
\hline Mortality & $0(0.0 \%)$ & $0(0.0 \%)$ & 0.496 \\
\hline
\end{tabular}

${ }^{*}$ Significant $(p<0.05)$ associations.

significance level used was $p<0.05$. The data were analyzed with Statistical Package Stata 12.0 (Stata Corporation, College Station, TX).

\section{Results}

3.1. Study Population. A total of 303 infants with a diagnosis of acute bronchiolitis were evaluated. Of the 303 included patients, 176 (58.1\%) were males, and the median IQR age was $3.0(1.0-7.0)$ months. The age group distribution was $203(67.0 \%)$ less than 6 months, $92(30.4 \%)$ between 6 and 12 months, and the remaining $8(2.6 \%)$ between 13 and 24 months. Regarding the presence of a history of personal or familiar atopic diseases, 45 (14.9\%) patients had a history of parental asthma and 13 (4.3\%) a previous diagnosis of atopic dermatitis. All the related demographic and clinical variables, according to the appropriateness of bronchiolitis use of diagnostic tests and treatment, are presented in Table 1.

3.2. Use of Diagnostic Tests. The use of diagnostic tests in the study period was as follows: hemogram in 159 (52.5\%) patients, C-reactive protein in $52(17.2 \%)$, procalcitonin in 6 (2.0\%), chest X-ray in 209 (69.0\%), and tests for detection of respiratory viruses in 207 (68.3\%).

3.3. Pharmacologic Therapy. Rates of use of medications were as follows: inhaled or nebulized beta 2 agonists in $271(89.4 \%)$ patients, inhaled or nebulized anticholinergics in 30 (9.9\%), nebulized epinephrine in 94 (31.0\%), inhaled corticosteroids in 53 (17.5\%), systemic corticosteroids in 52 (17.2\%), antibiotics in 89 (29.4\%), and nebulized hypertonic saline in $48(15.8 \%)$.

3.4. Care or Bronchiolitis-Severity Parameters. With respect to the care or the disease-severity parameters, the median (IQR) of the length of hospital stay was 4.0 (3.0-5.0). Out of the total of 303 patients included in the study, $98(32.3 \%)$ had a continued need for oxygen therapy at home, $4(1.3 \%)$ required admission to the PICU, 1 (0.3\%) required endotracheal intubation, and none died due to the respiratory disease.

3.5. Inappropriate Use of Diagnostic Tests and Treatment of Bronchiolitis. Based on the above-mentioned definition, it was considered that $216(71.3 \%)$ patients experienced an inappropriate use of diagnostic tests and treatment of bronchiolitis. This was mainly due to the use of beta 2 agonists in 271 (89.4\%) patients, a lack of the use of nebulized hypertonic saline in 252 (83.2\%), the use of diagnostic tests such as chest X-rays in 209 (69.0\%), and a hemogram in 159 $(52.5 \%)$. When compared with patients with an appropriate use of diagnostic tests and treatment of the disease, patients with an inappropriate use of diagnostic tests and treatment had a significantly longer hospital stay (4.0 [3.0-6.0] versus $3.0[2.0-4.0], p<0.001)$ and a significant higher isolation rate of respiratory syncytial virus (RSV) $(44.0 \%$ versus $23.0 \%$, $p<0.001)$. The remaining demographic and clinical 
TABLE 2: Predictors of inappropriate diagnosis and treatment of bronchiolitis through multivariate analysis.

\begin{tabular}{lcr}
\hline Variable & $\begin{array}{c}\text { Odds ratio (OR) } \\
(95 \% \text { CI })\end{array}$ & $p$ value \\
\hline Age & $0.95(0.84-1.07)$ & 0.378 \\
Number of siblings & $1.92(1.13-3.26)$ & $\mathbf{0 . 0 1 5}$ \\
Number of days with respiratory symptoms & 1.00 & - \\
$\quad$ Less than 24 hours & $1.19(0.23-6.24)$ & 0.834 \\
$\quad$ Between 24 and 72 hours & $2.93(0.68-12.69)$ & 0.150 \\
$\quad$ More than 72 hours & $4.02(0.96-16.90)$ & 0.057 \\
Presence of at least one underlying disease condition & $2.34(1.78-30.74)$ \\
Previous use of oxygen therapy & $5.30(1.14-24.79)$ & 0.518 \\
Personal history or diagnosis of atopic dermatitis & $1.46(0.52-4.12)$ & $\mathbf{0 . 0 3 4}$ \\
Parental history of asthma & $1.48(1.08-2.03)$ \\
Length of hospital stay & & 0.470 \\
\hline
\end{tabular}

characteristics that were measured did not differ between the two groups of patients (Table 1).

\subsection{Predictors of Inappropriate Use of Diagnostic Tests and} Treatment of Bronchiolitis through Multivariate Analysis. Multivariate analyses were conducted to determine independent predictors of the outcome variable. The predictor variables included in the multivariate models were age, number of brothers and sisters, number of days with respiratory symptoms, presence of at least one underlying disease condition, personal history or diagnosis of atopic dermatitis, parental history of asthma, previous use of oxygen therapy, RSV isolation, and length of hospital stay. After controlling for these potential confounders, it was found that a personal history or diagnosis of atopic dermatitis (OR 5.30; CI 95\% $1.14-24.79 ; p=0.034$ ), length of hospital stay (OR 1.48; CI 95\% $1.08-2.03 ; p=0.015)$, and the number of siblings (OR 1.92; CI 95\% 1.13-3.26; $p=0.015$ ) were independent predictors of an inappropriate use of diagnostic tests and treatment of bronchiolitis in our sample of patients (Table 2). In the sensitivity analysis using cutoff values from 1 to 5 of the composite outcome score, predictors of "inappropriate use of diagnostic tests and treatment of bronchiolitis" did not change substantially (data not shown).

\section{Discussion}

The results of this study show that there was a high prevalence of an inappropriate use of diagnostic tests and treatment of bronchiolitis in infants hospitalized in a third-level teaching referral hospital. Participants with a personal history or diagnosis of atopic dermatitis, a longer hospital stay, and a greater number of siblings were at increased risk for inappropriate use of diagnostic tests and treatment.

We are confident that our results may improve the knowledge about patient and disease characteristics, which can serve as a basis for planning more targeted implementation strategies that could help to improve the rational use of diagnostic tests and medications that lack strong evidence that supports a recommendation for their routine use in bronchiolitis. Although we identified a set of predictors independently associated with an inappropriate diagnosis and management of bronchiolitis not previously reported in the literature, our results are in line with previous studies that have also identified disease accompanying conditions or disease characteristics themselves as predictors of the appropriateness of the treatment. Henao-Villada et al. identified the presence of an underlying disease condition and bronchiolitis caused by viruses other than respiratory syncytial virus (RSV) as independent predictors of an inappropriate diagnosis and treatment of bronchiolitis in a population of children admitted to the emergency department or hospitalized for the disease. Additionally, age was inversely correlated with the appropriateness of the treatment, suggesting that younger patients have a greater probability of being adequately diagnosed and treated [11]. In our study, we identified that a personal history or diagnosis of atopic dermatitis and a longer hospital stay also independently predicted an inappropriate diagnosis and management of the disease. Taken together, these findings would seem to suggest that the less typical or less pure form the disease has, the lower the probability of having an appropriate diagnosis and management. The typical patient with acute viral bronchiolitis is a small infant with a demonstrated infection by RSV, which usually requires a short-term stay when hospitalization is necessary [12]. For this reason, it is plausible to consider that there is a greater probability of requesting diagnostic tests or prescribing the use of medications not routinely recommended in CPGs when clinicians deal with patients with bronchiolitis without this typical clinical presentation. For example, clinicians could consider that an infant suffering from bronchiolitis with a personal history or diagnosis of atopic dermatitis has a high probability of future asthma symptoms and could be more prone to treat the bronchiolitis episode in the same manner as an asthma exacerbation episode. Likewise, clinicians treating an older infant with initial symptoms suggestive of bronchiolitis but with prolonged symptom duration may feel compelled to use diagnostic tests or to prescribe medications that are not routinely recommended in evidence-based CPGs, probably in order to consider the 
possibility of an alternative diagnosis, or even because of the pressure of parents who feel that their child is not getting better. Other studies have identified other factors unrelated to the disease or the patient himself as predictors of inappropriate management of bronchiolitis, such as the region, the community, and the type of university affiliation of the hospital where the infants were treated $[7,8]$. Nevertheless, these results are difficult to compare with ours, because we performed a single-center study. We found that a greater number of siblings was associated with an increased risk of inappropriate diagnosis and management of bronchiolitis; however the reason for this association is not entirely clear. A reasonable explanation for this association may be due to the fact that the number of siblings has been reported as an independent predictor for recurrent wheezing following acute bronchiolitis [13]. It is probable that some of these recurrent wheezing episodes have been diagnosed as acute viral bronchiolitis but have been treated as acute asthma exacerbations.

A number of potential limitations need to be considered. First, we used a nonvalidated definition of inappropriate diagnosis and treatment of bronchiolitis. Although we based this definition on the recommendations provided by a current evidence-based CPG, there is a gap in the literature for evaluating the actual appropriateness of bronchiolitis management for specific indications. For example, it has been reported that atopic dermatitis aggravates the allergic airways inflammation in patients with acute viral bronchiolitis [14]. Therefore, it is probable that treating patients with acute viral bronchiolitis and coexisting atopic dermatitis with corticosteroids could not be considered completely inappropriate. However, current available evidence does not support such a practice. Second, since this study was based on an EMR review, assessing the appropriateness of the diagnosis and management of bronchiolitis was based on information documented in the patient's EMR. It is possible that a patient may have had additional symptoms or findings that would have altered the appropriateness of the diagnosis and management of the disease. Third, patients diagnosed and treated in other settings (emergency department, outpatients) were not included in the present study, thus limiting its generalizability. Finally, as opposed to other studies in which the variability and the appropriateness of bronchiolitis management were determined in a representative sample of all Health Care Centers and all regions of the country [8], our study was limited to patients hospitalized at a single pediatric hospital. Therefore, our results need to be interpreted with caution because they could not be generalizable to all bronchiolitis patients. However, we are confident that our results represent an excellent initial step toward designing interventions that include a more rational approach to the diagnosis and treatment of patients with bronchiolitis.

\section{Conclusions}

In conclusion, the findings of the present study show that there was a high prevalence of the inappropriate use and overuse of medications that have insufficient evidence of effectiveness and the use of unnecessary diagnostic tests in a population of hospitalized infants with acute viral bronchiolitis. Patients with a personal history or diagnosis of atopic dermatitis, a longer hospital stay, and a greater number of siblings were at increased risk of inappropriate diagnosis and treatment. Future research on a validated definition of an inappropriate diagnosis and treatment of bronchiolitis should be undertaken.

\section{Conflicts of Interest}

The authors declare that there are no conflicts of interest regarding the publication of this paper.

\section{Acknowledgments}

The authors would like to thank Mr. Charlie Barret for his editorial assistance.

\section{References}

[1] S. Leader, K. Kohlhase, M. H. Pearlman, J. V. Williams, and W. A. Engle, "Recent trends in severe respiratory syncytial virus (RSV) among US infants, 1997 to 2000," Journal of Pediatrics, vol. 143, no. 5, pp. S127-S132, 2003.

[2] L. C. Paramore, V. Ciuryla, G. Ciesla, and L. Liu, "Economic impact of respiratory syncytial virus-related illness in the US: an analysis of national databases," PharmacoEconomics, vol. 22, no. 5, pp. 275-284, 2004.

[3] C. E. Rodriguez-Martinez, M. P. Sossa-Briceño, and R. AcuñaCordero, "Quality assessment of acute viral bronchiolitis clinical practice guidelines," Journal of Evaluation in Clinical Practice, 2015

[4] C. Ochoa Sangrador and J. González de Dios, "Overuse of bronchodilators and steroids in bronchiolitis of different severity. Bronchiolitis-study of variability, appropriateness, and adequacy," Allergologia et Immunopathologia, vol. 42, no. 4, pp. 307-315, 2014.

[5] C. E. Rodriguez Martinez and M. P. Sossa Briceno, "Costeffectiveness of chest $\mathrm{x}$-rays in infants with clinically suspected viral bronchiolitis in Colombia," Revista Panamericana de Salud Pública, vol. 29, no. 3, pp. 153-161, 2011.

[6] T. A. Florin, T. Byczkowski, R. M. Ruddy, J. J. Zorc, M. Test, and S. S. Shah, "Variation in the management of infants hospitalized for bronchiolitis persists after the 2006 American Academy of Pediatrics bronchiolitis guidelines," Journal of Pediatrics, vol. 165, no. 4, pp. 786-792.el, 2014.

[7] H. R. Acuna-Cordero R, D. Montano, S. Martinez, and C. Beltran, Factores asociados al manejo inapropiado de bronquiolitis aguda en ocho ciudades de Colombia. Iatreia; 27: 4-S, 2012.

[8] C. Ochoa Sangrador and J. González De Dios, "Management of acute bronchiolitis in spanish emergency wards: variability and appropriateness analysis (aBREVIADo project)," Anales de Pediatria, vol. 79, no. 3, pp. 167-176, 2013.

[9] A. Galindo, F. Mendivelso, F. Palencia, M. Vallejo, and H. Gaitan, "Using a short strategy for adapting Clinical Practice Guidelines (CPG): experience in Colombia," in Guidelines International Network (G-I-N) Conference, Melbourne, Australia, 2014.

[10] N. Gamba-Sanchez, C. E. Rodriguez-Martinez, and M. P. Sossa-Briceño, "Epidemic activity of respiratory syncytial virus is related to temperature and rainfall in equatorial tropical 
countries," Epidemiology and Infection, vol. 144, no. 10, pp. 20572063, 2016.

[11] R. Henao-Villada, M. P. Sossa-Briceño, and C. E. RodríguezMartínez, "Impact of the implementation of an evidencebased guideline on diagnostic testing, management, and clinical outcomes for infants with bronchiolitis," Therapeutic Advances in Respiratory Disease, vol. 10, no. 5, pp. 425-434, 2016.

[12] A. Nicolai, M. Ferrara, C. Schiavariello et al., "Viral bronchiolitis in children: a common condition with few therapeutic options," Early Human Development, vol. 89, no. 3, pp. S7-S11, 2013.

[13] L. Cifuentes, S. Caussade, C. Villagrán et al., "Risk factors for recurrent wheezing following acute bronchiolitis: a 12-month follow-up," Pediatric Pulmonology, vol. 36, no. 4, pp. 316-321, 2003.

[14] D. Marchand, N. Tayara, M.-L. Choukroun et al., "Atopic dermatitis aggrevates the allergic airways inflammation in acute viral bronchiolitis," Revue des Maladies Respiratoires, vol. 25, no. 9, pp. 1087-1093, 2008. 


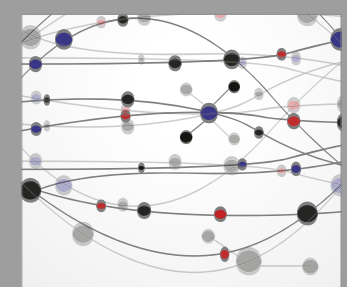

The Scientific World Journal
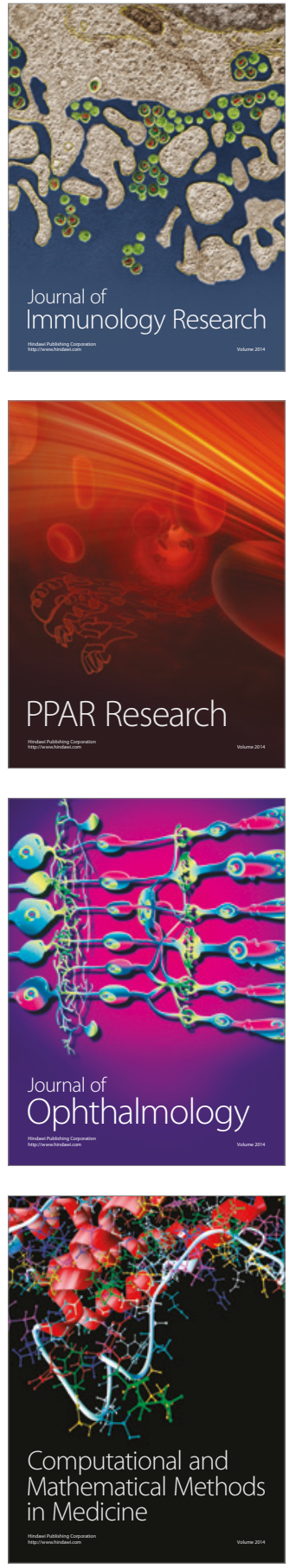

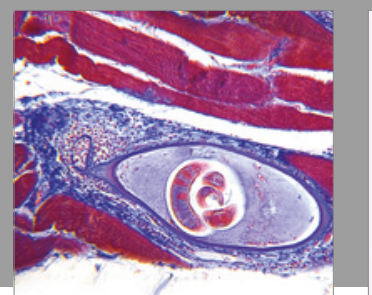

Gastroenterology Research and Practice
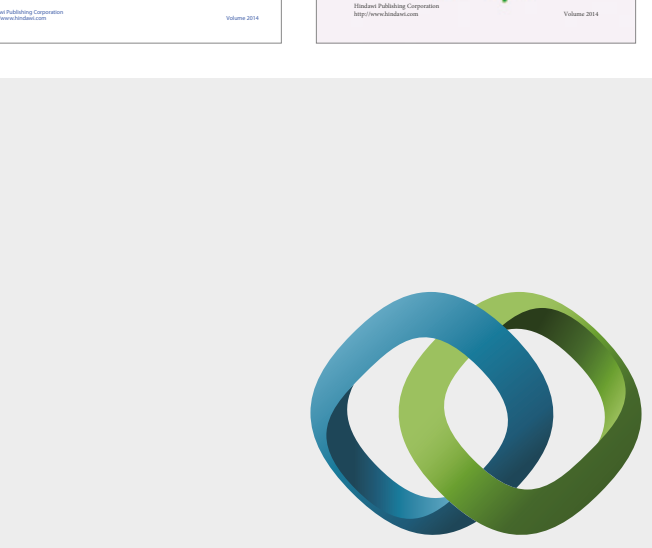

\section{Hindawi}

Submit your manuscripts at

https://www.hindawi.com
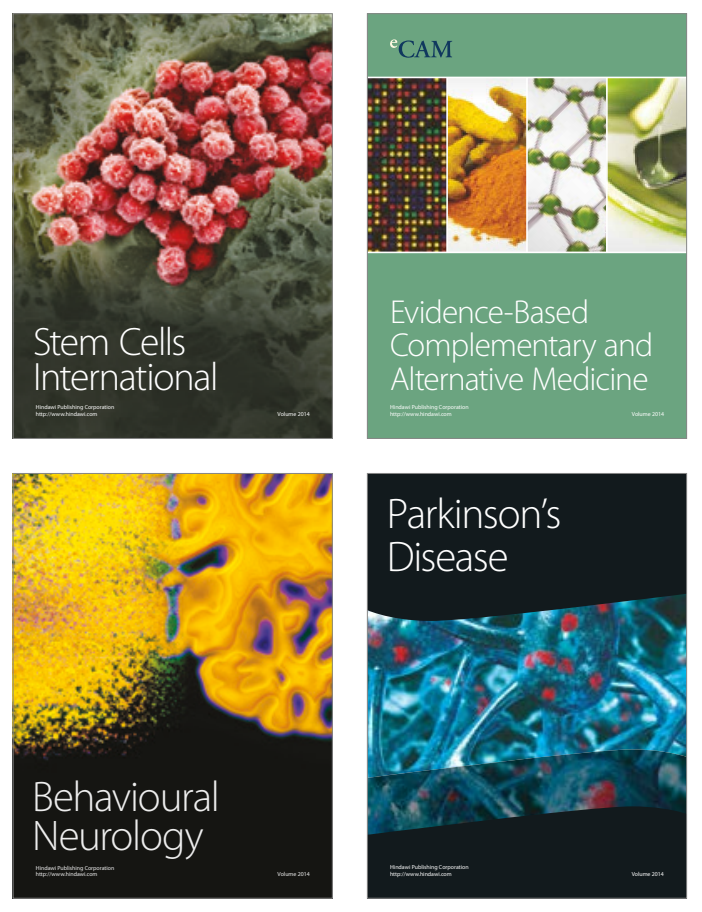
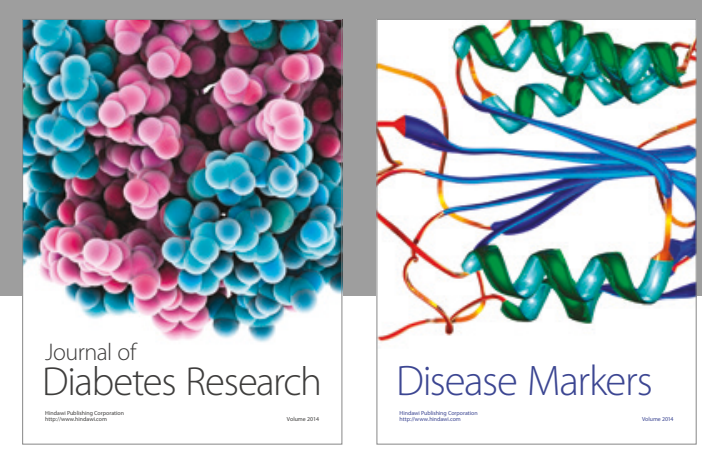

Disease Markers
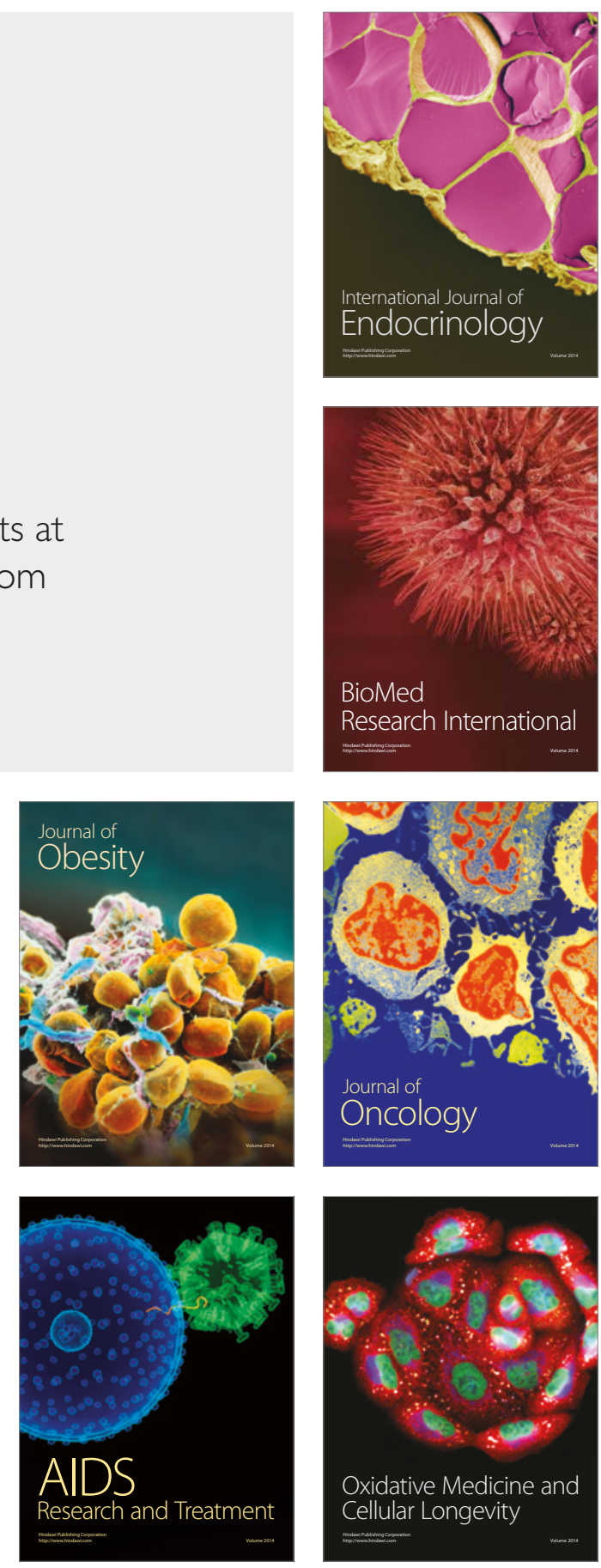